\title{
Review
}

\section{Impact of near infrared (NIR) spectroscopy and hyperspectral (HS) imaging system to predict physicochemical composition and quality attributes of meat: A review}

\author{
M. N. Uddin ${ }^{1}$ M. N. Hossain ${ }^{1}$, N. Z. Shoshe ${ }^{1}$, S. A. Toma ${ }^{1}$, S. A. Belal ${ }^{2}$, M. R. Akanda ${ }^{3}$, M. A. \\ Zaman $^{4}$, F. M. A. Hossain ${ }^{4}$, B. E. Maburutse ${ }^{5}$ \\ ${ }^{1}$ Department of Livestock Production and Management, Sylhet Agricultural University, Sylhet-3100, Bangladesh \\ ${ }^{2}$ Department of Poultry Science, Sylhet Agricultural University, Sylhet-3100, Bangladesh \\ ${ }^{3}$ Department of Pharmacology and Toxicology, Sylhet Agricultural University, Sylhet-3100, Bangladesh \\ ${ }^{4}$ Department of Dairy Science, Sylhet Agricultural University, Sylhet-3100, Bangladesh \\ ${ }^{5}$ Department of Animal Production Sciences and Health, Marondera University of Agricultural Sciences and Technology, \\ Zimbabwe
}

\section{Article History}

Received: 23 July 2021

Revised: 21 September 2021

Accepted: 24 September 2021

Published online: 30 September 2021

\section{*Corresponding Author}

M. N. Uddin, E-mail: uddinmn.alm@sau.ac.bd

\section{Keywords}

NIR spectroscopy, hyperspectral imaging, meat, chemical composition, quality

\section{A B S T R A C T}

In contrary to orthodox methods for determination of physicochemical composition and quality characteristic of meat, near infrared spectroscopy (NIRS) and hyperspectral imaging (HSI) systems are more reliable, prompt, simple and concurrent evaluation of abundant meat properties. This present review effort on the use of the NIRS and the HSI system to predict diverse meat properties, based on published literature in different years. The NIRS and the HSI exhibits a noticeable prospective to replace the expensive and laborious chemical analysis of meat composition mainly crude protein (CP), intramuscular fat (IMF), moisture, dry matter (DM), ash, collagen content, technological objective measurement $\left(\mathrm{pH}\right.$ value, color value, $\mathrm{L}^{*}, \mathrm{a}^{*}, \mathrm{~b}^{*}$, water holding capacity, warner-Bratzler shear force) and sensory characteristics (Tenderness, juiciness, chewiness, flavor, texture, odor and firmness). HSI system conglomerates imaging and spectroscopic technology is promptly getting field as a nondestructive, real- time recognition tool for food quality. The review notice that NIR revealed great potential to evaluate physicochemical properties of meat and to classify meat into quality classes based on meat quality traits for instances distinctive between feeding systems, discriminating fresh from-frozen-thawed meat and so on. Moreover, NIRS is less precise for evaluating various technological and sensory attributes of meat due to heterogeneity of meat samples and their preparation. Hence, further study is recommended to improve sensory and technological attributes by standardize the sample preparation and accuracy of referencing technique. In conclusion, the NIRS and the HIS are considered to be a very satisfactory system for swift meat evaluation.

(C) 2021 The Authors. Published by Society of Agriculture, Food and Environment (SAFE). This is an Open Access article distributed under the terms of the Creative Commons Attribution 4.0 License (http://creativecommons.org/licenses/by/4.0)

\section{Introduction}

Meat is a commonly consumed human diet throughout the world and greatly valued by the consumer due to its high nutritional value and contents of valuable nutrients such as protein, fat, vitamin and micronutrients (Verbeke et al., 2011). In this modern digital age, consumers have a more entrance to information; consequently they are more sensible about the meat that they eat particularly their quality, safety and authenticity (Papadopoulou et al., 2011). Quality and safety of human food are generally defined by physical aspects (i.e., texture, color, marbling, tenderness), chemical aspects (i.e., fat content, moisture, protein content, $\mathrm{pH}$, drip loss), and biological aspects (i.e., total bacterial count). Among many quality parameters, tenderness is considered as one of the most important characteristics that affects the eating quality of meat. Tenderness is the most important textural attributes that affecting consumer perception of eating quality of meat. Conventionally, assessment of food quality and safety involves human visual inspection, in addition to chemical or biological determination experiments 
which are monotonous, laborious, destructive, and occasionally naturally unfriendly. In the meat industry, quality evaluation and control still performed manually, which is tedious, laborious, costly, time consuming and subject to human error and inconsistency. Accordingly, the meat processing industry seeks non-contact, non-destructive, rapid, accurate and efficient analytical methods for fast evaluation of meat and meat products.

In compare to orthodox techniques for the fortitude of meat quality attributes, near infrared reflectance (NIR) spectroscopy is a sensitive, prompt and non-destructive analytical method with ease in sample preparation permitting a concurrent assessment of various meat properties (Osborne, 2000). NIR spectroscopy has been effectively applied to assessable determination of chief components (moisture, fat and protein) in meat and meat products as has been indicated in the review of (Prevolnik et al., 2004). In fact, NIR in the transmittance mode has been sanctioned by AOAC (Anderson, 2007) for the marketable analysis of moisture, fat and protein in meat and meat products using free and opensources software (FOSS) artificial neural network (ANN) prediction models. Furthermore, NIR efficiently used to judgment between frozen and unfrozen beef (Thyholt \& Isaksson, 1997), beef and kangaroo meat (Ding \& Xu, 1999) as well as broiler and local chicken (Ding \& Xu, 1999) (McDevitt et al., 2005). On the other hand, studies appraising NIR to predict technological and sensory parameters in meat and meat products show less consistency (Andrés et al., 2007) (Hoving-Bolink et al., 2005) (ČandekPotokar et al., 2006).

Hyperspectral imaging is a comparatively novel method that incorporates both imaging and spectroscopy in a single system, allowing acquisition of spatial and spectral information concurrently from an object. As a consequence, the technology delivers unexpected data, which can be analyzed to notice, pinpoint, and enumerate imaged objects in more detail, which otherwise cannot be succeeded with either imaging or spectroscopy alone. Associated with multivariate analyses, hyperspectral imaging has been successfully carry out quality evaluation of various agricultural and livestock food products including red meat and white meat quality (D. F. Barbin, Elmasry, Sun, Allen, et al., 2013), (Elmasry et al., 2011) (Kamruzzaman et al., 2012), (Kamruzzaman et al., 2011), (Kamruzzaman, ElMasry, et al., 2013), and poultry (Feng \& Sun, 2012); (Nakariyakul \& Casasent, 2009). For example, hyperspectral imaging has been utilized for predicting tenderness, $\mathrm{pH}$, color, and water-holding capacity (WHC) in beef (ElMasry et al., 2012, 2012a, 2012b), (ElMasry, Barbin, Sun, \& Allen, 2012; ElMasry, Kamruzzaman, Sun, \& Allen, 2012; ElMasry, Sun, \& Allen, 2012a; ElMasry, Sun, et al., 2012b), pork (D. F. Barbin, Elmasry, Sun, \& Allen, 2013) (D. Barbin et al., 2012) (D. F. Barbin et al., 2012) and lamb (Kamruzzaman, ElMasry, et al., 2012a; (Kamruzzaman, Sun, et al., 2013). It has also been used to determine proximate analysis of mainly moisture, fat, and protein in beef (ElMasry et al., 2013), lamb (Kamruzzaman, ElMasry, Sun, \& Allen, 2012c), and pork (D. F. Barbin, Elmasry, Sun, \& Allen, 2013) (D. F. Barbin, Elmasry, Sun, Allen, et al., 2013). Therefore, this review paper pinpoint an overview of published research article that dealt with the relative impact of NIR and HIS to assess the chemical composition and quality attributes of meat from different food animal species.
Back ground history of near infrared reflectance (NIR) Spectroscopy

The innovation of near-infra red energy is accredited to Frederick William Hereschel in the 1800. He was an English astronomer working to determine which color in the visible spectrum delivered heat from the sun. The initial industrial practice launched in the 1950s. In its primordial phase NIR was used only as an add-on parts to additional optical devices that cast-off other wavelengths for instance ultraviolet, visible or mid infra-red spectrometers. Many authors have indicated that NIRS tool is a fast, simple, profitable, time consuming and an eco-friendly analytical device. After its primary use in 1970 by an assembly controlled by Norris for evaluating agricultural food specimen, at present, NIR spectroscopy is practice for the rapid estimation in a widespread variety of food materials comprising its increasing acceptance in meat quality evaluation. Communal solicitations with meat consist not only of the measureable assessment of biochemical composition, such as fat and protein, but also textural attributes, such as tenderness and hardness (Liu et al., 2003). NIR spectroscopy is the measurement of the wave length and intensity of the absorption of near-infrared light by a sample. It is a sort of molecular vibrational spectroscopy that pays for electromagnetic photon energy (hv) in the energy range of $2.65 \times 10-19$ to $7.96 \times 10-20 \mathrm{~J}$, which resemble to the wavelength range of $800 \mathrm{~nm}$ to $2500 \mathrm{~nm}$ (wave numbers: 13,300 to $4000 \mathrm{~cm}-1$ ) (Pasquini, 2003).

\section{The basic working principles and mode of spectra measurement by NIR spectroscopy}

The basic principles of NIR spectroscopy comprise the creation, record and analysis of spectra rising from the association of electromagnetic radiation with the biological substance (Techniques \& Food, 2018). Electromagnetic radiation emerges from NIR spectroscopy by interacting with a specimen may be absorbed, reflected or transmitted. Hence, there are diverse manners for NIR spectroscopy spectra measurement based on different utilization. But in normal sense NIR spectroscopy has couple of modes namely reflectance and transmittance. In the reflectance method, NIR electromagnetic energy is absorbed against the surface of the sample. The electromagnetic energy dispersed off the surface is dignified by a suitable optical sensor (typically made of lead sulphide). Register the response of definite molecular bonds (for example, O-H; N-H; C = O) to NIR radiation, produces a spectrum that may be attributes of a specimen and may represent as a "fingerprint" (Woodcock et al, 2008). That spectrum expresses the physiochemical information of any employed organic molecules and may consequently produce worthwhile data about the configuration of a product (Liu et al., 2000). By collecting all of this data, spectra permit us to assess, discover and choice a population of specimen formerly we decide what we should measure by established method. This is a significant research characteristic of spectroscopic methods (Alomar et al., 2003). Principal component of NIR spectrometer: NIR spectrometer instrument primarily contains of light source, beam splitter system (wavelength selector), sample holder, optical detector, and data processing analyzed system. Each of these key components can have diverse backgrounds and possessions for that reason it is conceivable to categorize the instruments on basis of the features of the parts which constitute the instrument itself. NIR spectrometer produces two types of NIR radiation namely thermal radiation and 
non-thermal radiation. The thermal group contains the quartz halogen lamps and the Nernst filament. The non-thermal group comprises of light-emitting diodes (LED), laser diodes and lasers which are the main source of light, which consist of discharge lamp. Spectrophotometers can be differentiated in to discrete-wavelength spectrophotometers and continuous spectrum NIR on the basis of wavelength selection. The discrete-wavelength spectrophotometers irradiate a sample with only a few wavelengths selected using filters or lightemitting diodes (LEDs). The continuous spectrum may comprise a spreading discordant or an interferometer. The specimen must be pulverized into a regular satisfactory powder for meaningful measurements. NIR energy transects the surface of a sample due to the transmittance approach. A portion of this energy is transferred through the sample and exists from the end of the sample. A silicon detector is used to measure this exiting scattered light (Du \& Sun, 2004). Figure-1 illustrated the principle components of NIR spectroscopy.

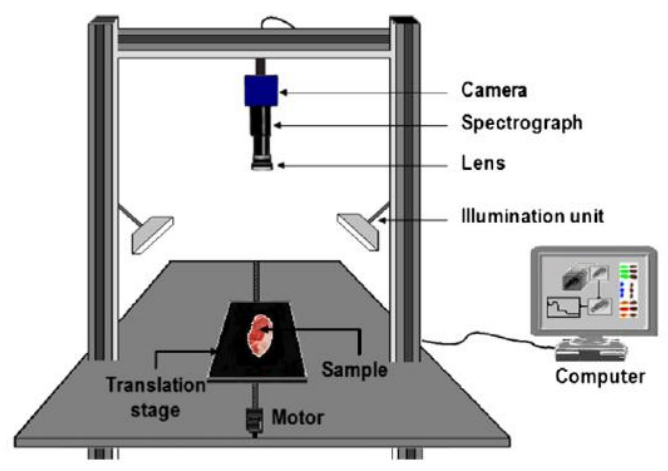

Figure 1. Schematic diagram of the main components of the NIR hyper spectral imaging system (Adapted with permission Kamruzzaman et al., 2012).

\section{Positive and negative aspects of NIR spectroscopy}

NIR spectroscopy comprises speediness of action, simplicity for specimen preparation, multiplicity for analysis of single spectrum and non-invasive to specimen, those are the four vital advantages. Other advantages are - It is a non-invasive, non-destructive, cost effective technique for either estimate of food animal's meat quality or authentication of addedvalue meat products as well as it is easy and convenient to collect near-infrared spectra from intact meat samples of various animal species (Prieto et al., 2017). It needs minimal or no sample preparation. Solid samples can be directly measured with little pretreatment, or no pretreatment. NIR spectroscopy is suitable for testing the chemical composition and properties of a range of biological materials, including meat. NIR spectroscopy also used to predict the physical parameters of an object likely density, viscosity and particle size. NIR spectroscopy instrumentation is most suitable for use in process control at production plants due to its have no moving parts. NIR spectroscopy fiber optic provides robust, strong sensors for at-line, on-line and in-line analysis to control process.

\section{Negative aspect of NIR spectroscopy}

NIR spectroscopy measurements are barely particular, so chemo metric methods have to be applied to model data from which to collect applicable data. There are no perfect models to receive the communication between NIR light and matter. Consequently, calibration is virtuously pragmatic in many cases. Correct, vigorous calibration models are problematic to achieve as their structure involves via a large ample quantity of samples to incorporate all distinctions in physical and/or chemical attributes. The NIR spectroscopy is not very complex, so generally it can be useful merely to key constituents. As NIR spectroscopy is a virtual method, to design models practicing it entails previous information of the value for the object parameter, which must be earlier determined applying a reference method. The development of NIR models needs extensive speculation, which can, however, be remunerated by shifting calibrations from the controlling equipment to numerous slaves. In spite of imperative developments in modern years, no exact methodology for this has yet expanded widespread reception. Moreover, NIR spectroscopy still has some limitations and challenges for online application under industrial environmental considerations (Prieto et al., 2017).

\section{NIR spectroscopy utilization to predict chemical composition, objective attributes sensory characteristics and meat categorization of different food animals}

Evaluation of meat constituents is crucial due to its relationship with overall quality characteristics and palatability attributes, freshness, and effect on end user health (Prieto et al., 2017). NIR spectroscopy has ascertained to be an extremely consistent method for the assessment of the content of all deliberated chemical components inside single muscle and collective group of different meats and meat products that is originated from different animal species. For instances in beef, the maximum researcher has been paid more concentration in their work by using NIR spectroscopy to predict the chemical composition and tenderness of red meat, that is the utmost influential sensory attributes of beef for consumers preference to acceptance of beef (Leroy et al., 2004) (Liu et al., 2003). For pork, the most of the research was carried out to assess the prospect of technological quality of meat or its indicators namely, $\mathrm{pH}$, water-holding capacity and chemical composition of meat, i.e. intramuscular fat, protein and moisture content (Meulemans et al., 2003). For lamb, the researcher used NIR spectroscopy to estimate the chemical composition (Intramuscular fat, Protein content and Moisture) and some other quality traits of meat (Cozzolino et al., 2000). For poultry meat, (Cozzolino et al., 1996) applied NIR spectroscopy to predict physical, color and sensory attributes of meat.

Recently, NIR spectroscopy device deals diverse statistical regression approaches to formulate standardizations (equations); multiple linear regression (MLR), partial and modified partial least square (MPLS), principal component analysis (PCA) and also a new method that permits for nonlinear relationship, neural networks (i.e. Win ISI, Infra-soft International, LLC, 2000). Cross validation technique was used to predict the accuracy of the software used in modern NIR spectroscopy instrument. Cross validation is a technique where every specimen in the calibration is anticipated; anticipate groups are prepared by eliminating one or more specimen from standardization set and the process is continual up to all specimens have had single turn in an estimate set. Error of cross-validation thus characterizes a factual evaluation of the prediction accuracy. The prediction aptitude of NIRS is usually arbitrated by statistical parameters: coefficient of determination $(R 2)$ and standard error (SE) of calibration and/or prediction. The capability of NIRS to assess biochemical configuration of meat was studied by a several of researcher. Different researchers used 
different NIR spectroscopy wave length spectra to predict chemical composition of different sorts of meat obtain from different food animals. Prieto et al., 2014 used 400-2498 nm wavelength NIR spectroscopy spectra to determine chemical composition of beef from homogenized meat samples. Prevolnik et al., 2010 used 400-2500 nm 400-2498 nm wavelength NIR spectroscopy spectra to predict chemical composition of pork muscle meat and muscles meat of others food animals at a raw state. Balage et al., 2015 used 400$1395 \mathrm{~nm}$ wavelength 400-2498 nm wavelength NIR spectroscopy spectra to detect intramuscular fat content in pork and beef. Su et al., 2014 used 1000-1800nm wave length 400-2498 nm wavelength NIR spectroscopy spectra to determine moisture, protein and intramuscular fat content of homozenized beef samples. So far, various calibration have been established for evaluating beef (Tøgersen et al., 2003) (Alomar et al., 2003), pork (Tøgersen et al., 1999; Brøndum et al., 2000b) lamb (Cozzolino et al., 2000) and poultry meat (Valdes and Summers, 1986; Renden et al., 1986; Abeni and Bergoglio, 2001).Though the maximum of the studies concentrated on evaluate one or more key constituents such as protein, fat and moisture (Cozzolino et al., 1996) (Cozzolino et al., 2000) (Tøgersen et al., 1999) few of them intended to predict collagen (Alomar et al., 2003) (Young et al., 1996), fatty acids (González-Martín et al., 2003) and ash (Alomar et al., 2003). The capacity of NIRS to estimate biochemical composition of meat from different animal origin collected from different studies by different researcher in different period of time presented as concise form in Table $1,2,3 \& 4$. On the basis of the study, the outcomes for estimate aptitude are also achieved on prediction sample set or by cross-validation on the equivalent, calibration sample set; in some studies only calibrations were made. According to the presented results from the summarizing table, we can sum up that the application of NIRS to estimate meat biochemical characters is mostly significant, as in the substance of the published studies high determination coefficients (above 0.80) were found. The maximum calibration and/or prediction correctness is stated for intramuscular fat content and fairly lesser for protein and moisture content. The described results for prediction of collagen content in meat using NIRS were much lesser; it could be owing to either weak sensitivity of NIRS to petty ingredients (Büning-Pfaue, 2003) or to the consistency of the reference method (colorimetric), which is occasionally evaluated for tissues low in collagen, like meat.

Table 1. Prediction Chemical compositions of meat by spectroscopic method arranged by animal origin.

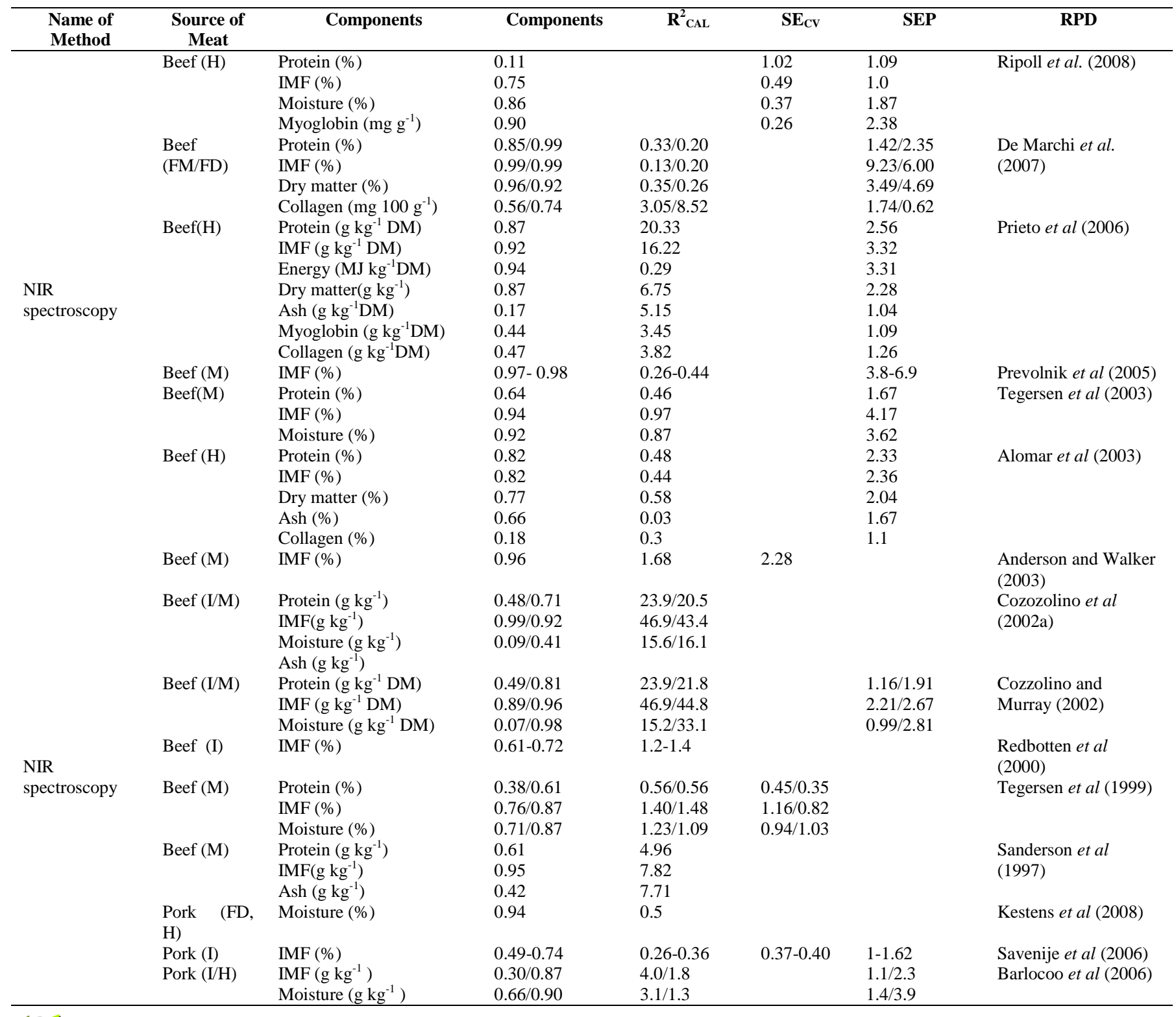


Uddin et al., 2021

\begin{tabular}{|c|c|c|c|c|c|c|c|}
\hline $\begin{array}{l}\text { Name of } \\
\text { Method }\end{array}$ & $\begin{array}{c}\text { Source of } \\
\text { Meat }\end{array}$ & Components & Components & $\mathbf{R}_{\text {CAL }}^{2}$ & $\mathbf{S E}_{\mathbf{C V}}$ & SEP & RPD \\
\hline & Pork (I/M) & $\begin{array}{l}\text { IMF (\%) } \\
\text { Protein (\% DM) } \\
\text { IMF (\% DM ) } \\
\text { Dry matter (\% DM) } \\
\text { Ash (\% DM) }\end{array}$ & $0.97 / 0 . .99$ & $0.34 / 0.23$ & & $5.3 / 8.3$ & Vijioen et al (2005) \\
\hline & Pork (I) & $\operatorname{IMF}\left(\mathrm{g} \mathrm{kg}^{-1}\right)$ & 0.35 & 3.6 & & & $\begin{array}{l}\text { Hoving-Bolink et al } \\
\text { (2005) }\end{array}$ \\
\hline & Pork (I) & Protein $(\%)$ & 0.69 & 0.0042 & 0.0042 & & Chan et al (2002) \\
\hline & & $\operatorname{IMF}(\%)$ & 0.76 & 0.0062 & 0.0062 & & \\
\hline & & Moisture (\%) & 0.8 & 0.0058 & 0.0063 & & \\
\hline & Pork (I) & $\begin{array}{l}\operatorname{IMF}(\%) \\
\text { Moisture (\%) }\end{array}$ & $\begin{array}{l}0.49 \\
0.21\end{array}$ & $\begin{array}{l}1.32 \\
1.13\end{array}$ & & $\begin{array}{l}1.44 \\
1.24\end{array}$ & $\begin{array}{l}\text { Bronndum et al } \\
\text { (2000a) }\end{array}$ \\
\hline & Pork (M) & Protein $(\%)$ & $0.38 / 0.61$ & $0.56 / 0.56$ & $0.45 / 0.35$ & & Tegersen et al (1999) \\
\hline & & $\operatorname{IMF}(\%)$ & $0.76 / 0.87$ & $1.40 / 1.48$ & $1.16 / 0.82$ & & \\
\hline & & Moisture (\%) & $0.71 / 0.87$ & $1.23 / 1.09$ & $0.94 / 1.03$ & & \\
\hline & Mutton & Protein (DM \%) & 0.98 & & 0.92 & 11.39 & Vijioen et al.(2007) \\
\hline & $(\mathrm{FD}, \mathrm{M})$ & IMF (\% DM) & 1.00 & & 0.43 & 28.46 & \\
\hline NIR & & $\operatorname{Ash}(\%)$ & 0.98 & & 0.38 & 3.40 & \\
\hline \multirow{8}{*}{ spectroscopy } & Lamb (I) & $\operatorname{IMF}(\%)$ & 0.84 & 0.41 & & 2.20 & Andres et al. (2007) \\
\hline & & Moisture (\%) & 0.67 & 0.69 & & 1.57 & \\
\hline & $\operatorname{Lamb}(\mathrm{I} / \mathrm{M})$ & Protein $\left(\mathrm{g} \mathrm{kg}^{-1} \mathrm{DM}\right)$ & $0.71 / 0.83$ & $8.8 / 5.5$ & & $1.41 / 2.18$ & Cozzolino and \\
\hline & & $\mathrm{IMF}\left(\mathrm{g} \mathrm{kg}^{-1} \mathrm{DM}\right)$ & $0.34 / 0.73$ & $8.1 / 4.7$ & & $1.09 / 1.81$ & Murray (2002) \\
\hline & & Moisture $\left(\mathrm{g} \mathrm{kg}^{-1} \mathrm{DM}\right.$ & $0.55 / 0.76$ & $15.5 / 10.3$ & & $1.25 / 1.89$ & \\
\hline & Lamb (I/M) & Protein $\left(\mathrm{g} \mathrm{kg}^{-1}\right)$ & $0.71 / 0.83$ & $8.8 / 5.5$ & & $1.41 / 2.18$ & Cozozolino et al \\
\hline & & $\operatorname{IMF}\left(\mathrm{g} \mathrm{kg}^{-1}\right)$ & $0.34 / 0.73$ & $8.1 / 4.7$ & & $1.09 / 1.83$ & $(2000)$ \\
\hline & & Moisture $\left(\mathrm{g} \mathrm{kg}^{-1}\right)$ & $0.55 / 0.76$ & $15.5 / 10.4$ & & $1.25 / 1.88$ & \\
\hline
\end{tabular}

$\mathrm{R}^{2}{ }_{\mathrm{CAL}}$ : Coefficient of determination of calibration, $\mathrm{SE}_{\mathrm{CV}}$ : standard error of cross validation, $\mathrm{SEP}$ : standard error of prediction, RPD: ratio performance deviation calculated as SD/SEP, M: minced, I: intact, H: homogenized, FM: fresh minced, FD: freeze-dried, IMF: intramuscular fat. a Coefficient of determination of cross validation. b Not reliable.

Table 2. Prediction of objective attributes in meat by Spectroscopy methods sorted by animal species (Bovine, Swine and Ovine).

\begin{tabular}{|c|c|c|c|c|c|c|c|}
\hline $\begin{array}{l}\text { Name of } \\
\text { Method }\end{array}$ & $\begin{array}{c}\begin{array}{c}\text { Source of } \\
\text { Meat }\end{array} \\
\end{array}$ & Components & $\mathbf{R}_{\text {CAL }}^{2}$ & $\mathbf{S E}_{\mathrm{CV}}$ & SEP & RPD & References \\
\hline \multirow{21}{*}{$\begin{array}{l}\text { NIR } \\
\text { spectroscopy }\end{array}$} & Beef $(\mathrm{H})$ & WHC, Press loss (\%) & 0.86 & & 1.34 & 1.76 & Ripoll et al. (2008) \\
\hline & \multirow{9}{*}{$\begin{array}{l}\text { Beef }(\mathrm{H}) \text { (Adult } \\
\text { steer/young } \\
\text { cattle) }\end{array}$} & WBSF $(\mathrm{kg})$ & 0.74 & & 1.06 & 1.44 & \\
\hline & & $\mathrm{pH}$ & $0.41 / 0.47$ & $0.06 / / 0.08$ & & $1.12 / 1.26$ & \multirow[t]{8}{*}{ Prieto et al. (2008a) } \\
\hline & & Color $\mathrm{L}^{*}$ & $0.59 / 0.87$ & $1.50 / 1.56$ & & $1.24 / 2.17$ & \\
\hline & & Color a* & $0.008 / 0.71$ & $1.15 / 1.58$ & & $0.98 / 1.58$ & \\
\hline & & Color $\mathrm{b}^{*}$ & $0.35 / 0.90$ & $1.08 / 1.46$ & & $1.16 / 2.51$ & \\
\hline & & WHC, Press loss (\%) & $0.48 / 0.58$ & $2.08 / 2.51$ & & $1.11 / 1.30$ & \\
\hline & & WHC, Drop loss (\%) & $0.20 / 0.26$ & $0.36 / 0.55$ & & $1.02 / 1.04$ & \\
\hline & & WHC, Cooking loss (\%) & $0.001 / 0.14$ & $1.61 / 2.45$ & & $0.97 / 1.03$ & \\
\hline & & WBSF $(\mathrm{kg})$ & $0.17 / 0.45$ & $1.02 / 1.62$ & & $1.07 / 1.18$ & \\
\hline & Beef (I) $(0 \mathrm{~min}$ & $\mathrm{pH}$ & 0.97 & 0.10 & & 3.17 & \multirow[t]{6}{*}{ Andres et al (2008) } \\
\hline & \multirow{5}{*}{$\begin{array}{l}\text { p. m,/60 min p. } \\
\text { m) }\end{array}$} & Color $\mathrm{L}^{*}$ & $0.85 / 0.82$ & $1.16 / 1.36$ & & $2.22 / 2.07$ & \\
\hline & & Color a* & $0.29 / 0.35$ & $1.09 / 1.28$ & & $1.14 / 0.90$ & \\
\hline & & Color $\mathrm{b}^{*}$ & $0.49 / 0.51$ & $0.75 / 0.99$ & & $1.17 / 1.37$ & \\
\hline & & WHC, Press loss (\%) & 0.20 & 0.08 & & 1.01 & \\
\hline & & WBSF $\left(\mathrm{kg} \mathrm{cm}^{-2}\right)$ & 0.65 & 2.67 & & 1.46 & \\
\hline & \multirow{2}{*}{ Beef (FM/FD) } & WHC, Drip loss (\%) & $0.10 / 0.04$ & $3.50 / 3.44$ & & $0.49 / 0.50$ & \multirow[t]{2}{*}{ De Marchi et al (2007) } \\
\hline & & WBSF $\left(\mathrm{kg} \mathrm{cm}^{-2}\right)$ & $0.08 / 0.20$ & $5.21 / 4.99$ & & $1.09 / 1.34$ & \\
\hline & \multirow[t]{4}{*}{ Beef (I) } & Color $\mathrm{L}^{*}$ & 0.25 & & & & \multirow[t]{4}{*}{ Hoving-Bolink et al (2005) } \\
\hline & & Color a* & 0.31 & 1 & & & \\
\hline & & Color $\mathrm{b}^{*}$ & 0.06 & 1.6 & & & \\
\hline \multirow{20}{*}{$\begin{array}{l}\text { NIR } \\
\text { spectroscopy }\end{array}$} & & WHC, Drip loss (\%) & 0.004 & 1 & & & \\
\hline & \multirow[t]{4}{*}{ Beef (I) } & Color $\mathrm{L}^{*}$ & 0.55 & & & & \multirow[t]{4}{*}{ Liu et al (2003) } \\
\hline & & Color a ${ }^{*}$ & 0.90 & & & & \\
\hline & & Color b* & 0.78 & & & & \\
\hline & & WBSF $(\mathrm{kg})$ & $0.17-0.72$ & & & & \\
\hline & \multirow[t]{6}{*}{ Beef (I) } & Color L* & $0.64-0.85$ & $1.53-2.39$ & & $1.63-2.55$ & \multirow[b]{7}{*}{ Cozzolino and Murray } \\
\hline & & Color a* & $0.19-0.49$ & $1.15-2.51$ & & $1.08-1.39$ & \\
\hline & & Color $\mathrm{b}^{*}$ & $0.44-.075$ & $0.77-1.54$ & & $1.30-1.95$ & \\
\hline & & WHC, Drop loss (\%) & $0.38-0.54$ & $0.82-0.99$ & & $1.24-1.46$ & \\
\hline & & WHC, Cooking loss (\%) & $0.25-0.47$ & $1.81-2.31$ & & $1.13-1.40$ & \\
\hline & & WBSF $(\mathrm{N})$ & $0.12-0.41$ & $7.68-11.19$ & & $1.06-1.30$ & \\
\hline & Beef $(\mathrm{I} / \mathrm{H})$ & $\mathrm{pH}$ & $0.81 / 0.90$ & $0.18 / 0.13$ & & $2.11 / 1.92$ & \\
\hline & Beef (I) & WBSF $(\mathrm{N})$ & 0.58 & 10.6 & & 1.48 & Venel et al (2001) \\
\hline & \multirow[t]{4}{*}{ Beef (I) } & WBSF $\left(\mathrm{kg} \mathrm{cm}^{-2}\right)$ & 0.69 & 1.37 & & & \multirow[t]{4}{*}{ Redbotten et al (2001) } \\
\hline & & 2 days p. $\mathrm{m}$ & 0.62 & 1.49 & & & \\
\hline & & 9 days p. m & 0.36 & 2.54 & & & \\
\hline & & 21 days p.m & & & & & \\
\hline & $\begin{array}{l}\text { Beef (I) } \\
\text { (Bull/Cow) }\end{array}$ & WBSF $\left(\mathrm{kg} / 10 \mathrm{~cm}^{-2}\right)$ & $0.35 / 0.12$ & $16.9 / 11.6$ & & & Redbotten et al (2000) \\
\hline & Beef (I) & WBSF (kg) & 0.67 & 1.3 & & & Park et al (1998) \\
\hline & Beef (I) & WBSF (kg) & $0.37-0.67$ & $1.50-2.10$ & & & Byrne et al (1998) \\
\hline
\end{tabular}


Uddin et al., 2021

\begin{tabular}{|c|c|c|c|c|c|c|c|}
\hline $\begin{array}{l}\text { Name of } \\
\text { Method }\end{array}$ & $\begin{array}{c}\begin{array}{c}\text { Source of } \\
\text { Meat }\end{array} \\
\end{array}$ & Components & $\mathbf{R}^{2}{ }_{\text {CAL }}$ & $\mathbf{S E}_{\mathbf{C V}}$ & SEP & RPD & References \\
\hline & \multirow{5}{*}{ Pork (I/M) } & $\mathrm{pH}$ & $0.59 / 0.64$ & $0.11 / 0.11$ & $1.3 / 1.4$ & & \multirow{5}{*}{$\begin{array}{l}\text { Cander-Potokar et al } \\
\text { (2006) }\end{array}$} \\
\hline & & Color $\mathrm{L}^{*}$ & $0.53 / 0.37$ & $2.9 / 3.2$ & $1.3 / 1.1$ & & \\
\hline & & Color a* & $0.55 / 0.65$ & $1.3 / 1.3$ & $1.4 / 1.4$ & & \\
\hline & & Color $\mathrm{b}^{*}$ & $0.76 / 0.71$ & $1.0 / 1.1$ & $1.6 / 1.4$ & & \\
\hline & & WHC, Drip loss (\%) & $0.52 / 0.69$ & $2.1 / 2.0$ & $1.4 / 1.5$ & & \\
\hline & \multirow[t]{5}{*}{ Pork (I) } & $\mathrm{pH}$ & $0.69-0.83$ & $0.04-0.06$ & $0.05-0.07$ & $1.14-1.28$ & \multirow[t]{5}{*}{ Savenije et al (2006) } \\
\hline & & Color $\mathrm{L}^{*}$ & $0.72-0.79$ & $1.39-1.65$ & $1.25-1.64$ & $1.59-2.16$ & \\
\hline & & Color a $^{*}$ & $0.58-0.77$ & $0.57-0.59$ & $0.67-0.74$ & $1.19-1.35$ & \\
\hline & & Color $\mathrm{b}^{*}$ & $0.46-0.66$ & $0.46-0.52$ & $0.42-0.52$ & $1.35-1.67$ & \\
\hline & & WHC, Drip loss (\%) & $0.31-0.55$ & $1.24-1.35$ & $1.14-1.42$ & $1.05-1.27$ & \\
\hline & Pork $(\mathrm{I} / \mathrm{H})$ & $\operatorname{WBSF}\left(\mathrm{n} . \mathrm{cm}^{-1}\right)$ & $0.64 / 0.38$ & $8.5 / 9.0$ & & $1.2 / 1.1$ & Barlocco et al (2006) \\
\hline & Pork (I) & WHC, Drip loss (\%) & $0.46-0.79$ & $0.85-1.35$ & & & Pedersen et al (2003) \\
\hline & \multirow[t]{7}{*}{ Pork $(\mathrm{H})$} & $\mathrm{pH}$ & 0.12 & 0.09 & 0.08 & 1.25 & \multirow[t]{7}{*}{ Meulemans et al (2003) } \\
\hline & & Color $\mathrm{L}^{*}$ & 0.62 & 2.53 & 4.74 & 1.08 & \\
\hline & & Color a ${ }^{*}$ & 0.40 & 1.22 & 1.87 & 1.07 & \\
\hline & & Color $\mathrm{b}^{*}$ & 0.38 & 1.18 & 1.34 & 1.12 & \\
\hline & & WHC, Cooking loss (\%) & $\mathrm{b}$ & $\mathrm{b}$ & $\mathrm{b}$ & $\mathrm{b}$ & \\
\hline & & WHC, Drip loss (\%) & 0.54 & 1.41 & 2.35 & 0.81 & \\
\hline & & $\operatorname{WBSF}(\mathrm{N})$ & $\mathrm{b}$ & $\mathrm{b}$ & $\mathrm{b}$ & $\mathrm{b}$ & \\
\hline & \multirow[t]{2}{*}{ Pork (I) } & Drip Loss (\%) & $0.51-0.55$ & 1.1 & & & \multirow[t]{2}{*}{ Geesink et al (2003) } \\
\hline & & $\operatorname{WBSF}(\mathrm{N})$ & 0.20 & & & & \\
\hline & \multirow{3}{*}{ Pork (I/H) } & Color $\mathrm{L}^{*}$ & $0.30 / 0.62$ & $5.3 / 4.5$ & & $1.06 / 1.92$ & \multirow{3}{*}{ Cozzolino et al (2003) } \\
\hline & & Color a* & $0.93 / 0.95$ & $1.3 / 1.3$ & & $1.62 / 1.62$ & \\
\hline & & Color $\mathrm{b}^{*}$ & $0.60 / 0.27$ & $1.1 / 1.5$ & & $1.36 / 1.07$ & \\
\hline & \multirow[t]{4}{*}{ Pork (I) } & $\mathrm{pH}$ & 0.29 & 0.16 & 0.16 & 1.23 & \multirow[t]{4}{*}{ Chan et al (2002) } \\
\hline & & WHC, Press loss $\left(\mathrm{cm}^{2} \cdot \mathrm{g}^{-1}\right)$ & 0.34 & 2.75 & 2.45 & 1.40 & \\
\hline & & WHC, Cooking Loss (\%) & 0.23 & 0.05 & 0.06 & 1.07 & \\
\hline & & $\operatorname{WBSF}(\mathrm{N})$ & 0.17 & 13.8 & 14.42 & & \\
\hline & Pork (I) & $\mathrm{pH}$ & 0.33 & 0.074 & & $\mathrm{~b}$ & Josell et al (2000) \\
\hline & Pork (I) & WHC, Drip loss (\%) & 0.71 & 1.8 & & 1.75 & Forrest et al (2000) \\
\hline & \multirow{2}{*}{ Pork (I) } & WHC, Press loss (\%) & 0.38 & 16.01 & & 1.27 & \multirow{2}{*}{ Brendum et al (2000a) } \\
\hline & & WHC, Drip loss (\%) & 0.41 & 2.43 & & 1.32 & \\
\hline & Pork $(\mathrm{I} / \mathrm{H})$ & $\mathrm{pH}$ & $0.62 / 0.59$ & $0.08 / 0.10$ & & $1.66 / 1.59$ & Andersen et al (1999) \\
\hline & Lamb (I) & $\mathrm{pH}$ & $0.07-0.32$ & $0.16-0.26$ & & $0.90-1.22$ & Andres et al (2007) \\
\hline
\end{tabular}

Foot Note: $\mathrm{R}^{2}{ }_{\mathrm{CAL}}$ : Coefficient of determination of calibration, $\mathrm{SE}_{\mathrm{CV}}$ : standard error of cross validation, SEP: standard error of prediction, RPD: ratio performance deviation calculated as SD/SEP, I: intact, H: homogenized, M: minced, FM: fresh minced, FD: freeze-dried, RM: raw meat; CM: cooked meat, p.m.: post-mortem, WBSF: Warner-Bratzler shear force, WHC: water holding capacity. a Coefficient of determination of cross validation. $b$ Not reliable.

Table 3. Prediction of Sensory Characteristics of meat by Spectroscopy sorted by animal origin.

\begin{tabular}{|c|c|c|c|c|c|c|c|}
\hline $\begin{array}{l}\text { Name of } \\
\text { Method }\end{array}$ & $\begin{array}{c}\begin{array}{c}\text { Source of } \\
\text { Meat }\end{array} \\
\end{array}$ & Components & $\mathbf{R}_{\text {CAL }}^{2}$ & $\mathbf{S E}_{\mathbf{C V}}$ & SEP & RPD & References \\
\hline \multirow{31}{*}{$\begin{array}{l}\text { NIR } \\
\text { spectroscopy }\end{array}$} & \multirow{3}{*}{ Beef } & Juiciness & 0.54 & 0.61 & & 1.54 & Ripoll et al. (2008) \\
\hline & & Tenderness & 0.98 & 0.35 & & 3.82 & \\
\hline & & Overall appraisal & 0.56 & 0.44 & & 1.58 & \\
\hline & \multirow[t]{2}{*}{ Beef } & Juiciness & 0.50 & & & & Liu et al (2003) \\
\hline & & Chewiness & 0.58 & & & & \\
\hline & \multirow[t]{7}{*}{ Beef } & Flavour & 0.08 & 0.33 & & 1.06 & Venel et al (2001) \\
\hline & & Juiciness & A & A & & $\mathrm{a}$ & \\
\hline & & Tenderness & 0.26 & 0.78 & & 1.17 & \\
\hline & & Firmness & 0.10 & 0.63 & & 1.05 & \\
\hline & & Texture & 0.14 & 0.59 & & 1.07 & \\
\hline & & Chewiness & 0.16 & 0.33 & & 1.09 & \\
\hline & & Acceptability & 0.21 & 0.43 & & 1.09 & \\
\hline & Beef $(4 \mathrm{~h} / 26 \mathrm{~h}$ & Juiciness & A & 0.66 & & & Redbotten et al (2000) \\
\hline & \multirow{2}{*}{ NIR Spectra) } & Tenderness & $0.26 / 0.14$ & 0.96 & & & \\
\hline & & Chewiness & $0.19 / 0.12$ & 1.13 & & & \\
\hline & \multirow[t]{4}{*}{ Beef } & Flavour & $0.06-0.26$ & $0.35-0.39$ & & & Byrne et al (1998) \\
\hline & & Tenderness & $0.28-0.52$ & $0.71-0.88$ & & & \\
\hline & & Texture & $0.28-.050$ & $0.38-0.45$ & & & \\
\hline & & Acceptability & $0.18-0.50$ & $0.46-0.56$ & & & \\
\hline & \multirow[t]{3}{*}{ Pork } & Color & 0.45 & 0.33 & 0.28 & & Chan et al (2002) \\
\hline & & Marbling & 0.32 & 0.75 & 0.68 & & \\
\hline & & Firmness & 0.32 & 0.36 & 0.36 & & \\
\hline & \multirow[t]{4}{*}{ Pork } & Flavour & $0.004-0.59$ & & $0.20-1.15$ & $0.8-1.4$ & Brendum et al \\
\hline & & Odor & $0.01-0.23$ & & $0.55-1.03$ & $0.78-1.07$ & $(2000 b)$ \\
\hline & & Taste & $0.003-0.881$ & & $0.28-0.62$ & $0.82-2.71$ & \\
\hline & & Aftertaste & 0.69 & & 0.54 & 1.67 & \\
\hline & \multirow[t]{5}{*}{ Lamb } & Flavour & 0.34 & 0.47 & & & Andres et al (2007) \\
\hline & & Abnormal flavor & 0.13 & 0.44 & & & \\
\hline & & Juiciness & 0.38 & 0.44 & & & \\
\hline & & Texture & 0.16 & 0.85 & & & \\
\hline & & Overall liking & 0.27 & 0.48 & & & \\
\hline
\end{tabular}

Foot Note: $\mathrm{R}_{\mathrm{CAL}}^{2}$ : Coefficient of determination in calibration, $\mathrm{SE}_{\mathrm{CV}}$ : standard error of cross validation, SEP: standard error of prediction, RPD: ratio performance deviation calculated as SD/SEP, RM: raw meat, CM: cooked meat, PCR: principal component regression, PLSR: partial least-squares regression, MPLSR: modified partial least-squares regression, p: number of factors used to perform the calibration models. a Not reliable. 
Table 4. Classification of meat by Spectroscopy sorted by animal origin.

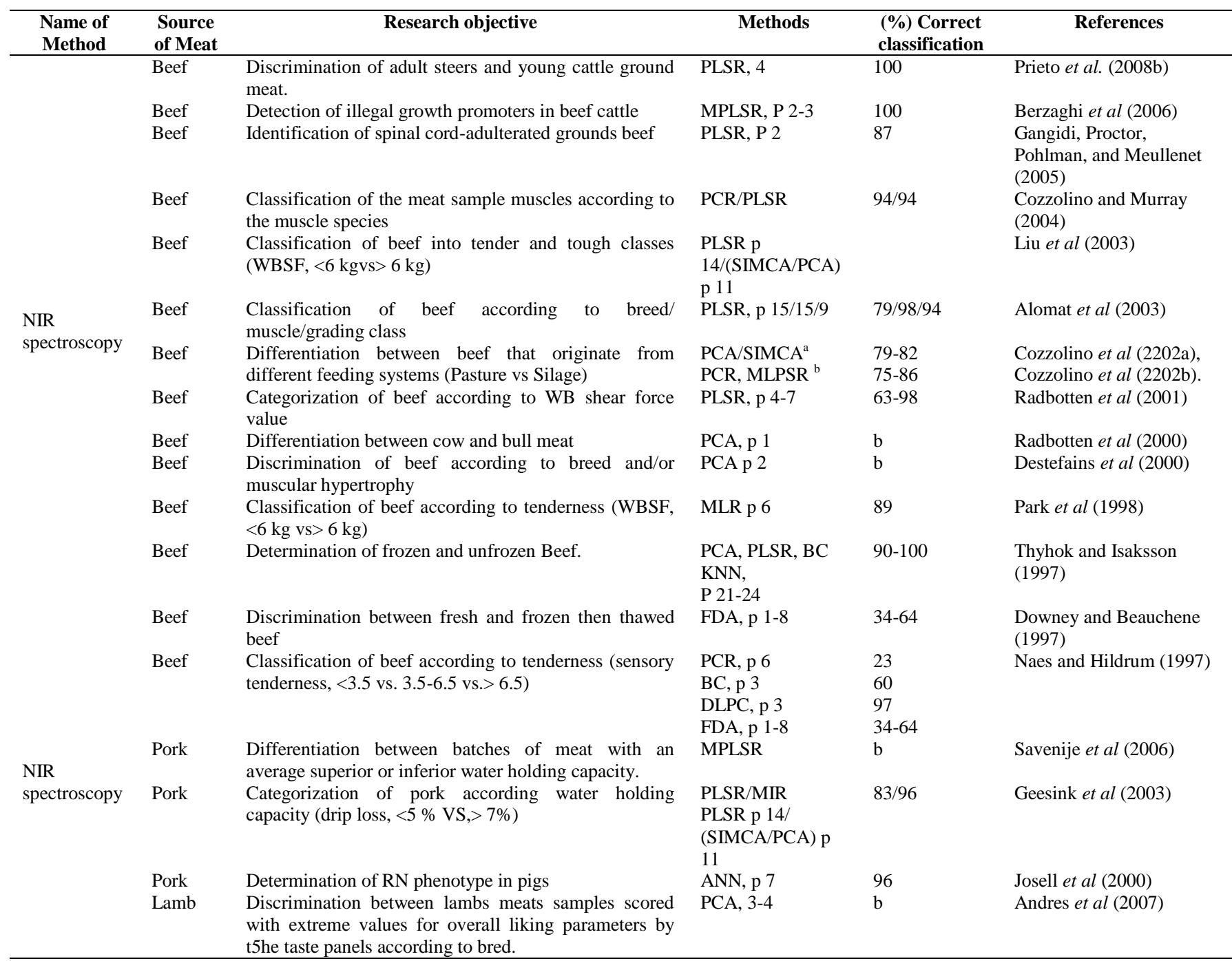

Foot Note FA: fatty acid, PCR: principal component regression, BC: Bayes classification, DLPC: distance-based discrimination for local principal components, FDA: factorial discriminant analysis, PCA: principal component analysis, PLSR: partial least-squares regression, KNN: K nearest neighbour, MLR: multilinear regression, CDA: canonical discriminant analysis, MPLSR: modified partial least-squares regression, ANN: artificial neural network, SIMCA: soft independent modelling of class analogy, p: optimum number of wavelengths or factors used in the regression equation. a Not reliable classification. b Acceptable categorisation.

\section{Fundamentals of hyper spectral imaging}

Hyperspectral imaging is one of the utmost favorable procedures presently explored for quality appraisal purposes in various sorts of applications. Hyperspectral imaging procedures convey spatial information, as regular imaging systems, in conjunction with spectral information for every pixel in the image. The chief benefit of the hyperspectral imaging system is its capability to integrate both spectroscopy and imaging techniques not only to create a direct assessment of diverse constituents concomitantly but also to pinpoint the spatial distribution of such constituents in the tested products. In its plenty applications, the hyperspectral imaging technique has revealed its potential in meat quality assessment (Naganathan et al., 2008) (Qiao et al., 2007) poultry carcass. Spectroscopy is one of the core optical procedures that has been efficiently executed in quality assessment of different types of meat such as pork (Hoving-Bolink et al., 2005); (Barlocco et al., 2006), beef (Ripoll et al., 2008), lamb (Andrés et al., 2007). The word "hyperspectral imaging" was first coined by Goetz et al (1985) during remote sensing activities (that means the observation of an object by a device without bodily interaction) to create an uninterrupted identification of external constituents in the form of images. The hyperspectral period commenced with airborne mineral mapping in the late 1970s and early 1980S. The invention of the first charge-coupled device in 1969 by George Smith and Willard Boyle was a fundamental element in affecting hyperspectral technology advancing (Gomez, 2002). The advancement happened in hyperspectral imaging technique during 1980s and 1990s.

\section{The basic working principle of hyperspectral imaging system}

Hyperspectral imaging can be defined as a concurrent acquisition of three-dimensional images in many spectrally adjacent bands measured from a remotely functioned platform (Schaepman, 2007). The basic principle of hyperspectral imaging is constructed on the circumstance that all materials, due to the variance of their biochemical conformation and natural physical structure, reflect, scatter, absorb, and release electromagnetic energy in unique patterns at definite wavelengths. This characteristic is termed spectral signature or spectral fingerprint, or simply the spectrum. A spectral signature is a distinctive attribute of an object. For a particular material, if the percentage of 
reflectance (also absorbance or transmittance) is schemed against wavelengths, the resulting curve is mentioned to as the spectral signature for the material. Each material has a distinct spectrum that expresses about its chemical composition (ElMasry et al., 2012). Basically, the spectral signature can be used to distinctively describe, recognize, and differentiate between classes/types of any particular materials in each pixel of the image. When the spectral data are properly processed, it is feasible to automatically detect the position of features that display exact spectral signatures and to map the gradient and spatial distribution of definite attributes.

There are three approaches to produce hyperspectral image established on the method by which spatial information is acquired as (I) point scanning / whiskbroom imaging (image is acquired point by point); (II) line scanning/ push broom (image is scanned line by line along one axis and (III) wavelength scanning/ area scanning (image of the whole object/area is acquired at a single wave length at a time.

\section{Components of hyperspectral imaging system}

The typical push broom hyperspectral imaging systems consist of a two-dimensional detector complementary metal Oxide Semiconductor (CCD or CMOS) camera, a spectrograph coupled with C-mount lens, an illumination unit, and a computer equipped with image acquisition software. Figure.-2 illustrated the principle components of hyperspectral imaging system.

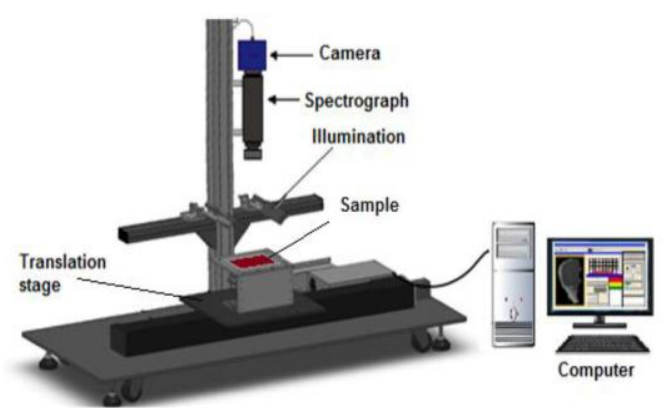

Figure 2. Schematic diagram of the main components of hyperspectral imaging system (Adapted with permission Kamruzzaman et al., 2012).

\section{Application of hyperspectral imaging system}

Hyperspectral imaging techniques have received a considerable attention for the quality of and safety assessment of meat, and has been successfully implemented for predicting tenderness and WHC in beef, classification and prediction of marbling in pork, contaminants and tumour detection in chicken and assessment of water and fat contents in fish fillets.

There are a number of areas for which hyperspectral imaging holds great potentials in quality evaluation of meat and meat products as shown in table 5,6 and in Figure 3. Meat is a usually consumed human diet all over the world and extremely valued by the consumer, primarily due to its valuable nutrients such as protein, fat, vitamin and micronutrients. Consumers are now more alarmed about the meat that they eat, and thus pay more awareness in terms of high quality, safety, and authenticity (Papadopoulou et al., 2011). The technique has been developed to conglomerate imaging and spectroscopy techniques in a solitary system to obtain both spatial and spectral information instantaneously. Because of the collective features of imaging and spectroscopy, hyperspectral imaging can be used to identify physical and symmetrical characteristics such as colour, size, shape and texture through image feature extraction as well as chemical configuration such as water, fat, protein, and other hydrogen-bonded components through spectral analysis (ElMasry et al., 2011). Hyperspectral imaging techniques have confirmed to be influential apparatuses for quantitative and qualitative analyses of a widespread range of materials for a large number of agricultural products (Feng \& Sun, 2012) (Shaarani et al., 2006). In specific, several studies have been accomplishing using hyperspectral imaging for red meat quality assessment. Their applications comprise the examination of tenderness, chemical composition, color, $\mathrm{pH}$ and drip loss in beef (ElMasry et al., 2011b, 2012a, 2012b; Naganathan et al., 2008a, 2008b; Cluff et al., 2008; Wu et al., 2012), classification, grading, prediction of quality and sensory characteristics and chemical configuration in pork (Barbin et al., 2012a; 2012b, 2012c, Qiao et al., 2007a, b,c), muscle discrimination, prediction of quality and sensory attributes, tenderness, chemical conformation and detection of authenticity and adulteration in lamb (Kamruzzaman et al., 2011, 2012a, 2012b, 2012c, 2013a, 2013b). Tenderness is one of the utmost significant textural characteristics that influence the eating quality of meat. It has been accepted as a vital quality factor that has considerable effect over customer pleasure as it is positively interrelated with juiciness and taste (Naganathan et al., 2008a). Discrepancies in meat tenderness have led to reduce in customer satisfaction and consequently in market shares (Naganathan et al., 2008b). Ensuring tenderness would rise confidence in consumers and stimulate frequent purchases. Numerous studies have revealed that consumers are eager to pay more for an assured tender meat (Shackelford et al., 2001). Preferably tenderness would be measured by a consumer panel as eventually the customer must be pleased.

Table 5. Applications of spectral imaging system in quality evaluation of beef and pork.

\begin{tabular}{|c|c|c|c|c|c|}
\hline Name of Method & $\begin{array}{l}\text { Source of } \\
\text { Meat }\end{array}$ & Application & $\begin{array}{l}\text { Wavelength range } \\
(\mathbf{n m})\end{array}$ & $\begin{array}{c}\text { Imaging } \\
\text { mode }\end{array}$ & References \\
\hline \multirow{9}{*}{$\begin{array}{l}\text { Hyperspectral imaging } \\
\text { technique. }\end{array}$} & Beef & Predicting microbial spoilage & $400-1100$ & Reflectance & Peng et al (2009) \\
\hline & Beef & Tenderness prediction & $400-1100$ & Reflectance & Peng and $W u(2008)$ \\
\hline & Beef & Tenderness prediction & $900-1700$ & Reflectance & $\begin{array}{l}\text { Naganathan et al } \\
(2008 \mathrm{~b})\end{array}$ \\
\hline & Beef & Tenderness prediction & $400-1000$ & Reflectance & $\begin{array}{l}\text { Naganathan et al } \\
\text { (2008a) }\end{array}$ \\
\hline & Beef & Tenderness prediction & 496-1036 & Reflectance & Cluff et al (2008) \\
\hline & Pork & Detection of total viable count of bacteria. & $400-1100$ & Reflectance & Peng and Wang (2008) \\
\hline & Pork & Determination of drip loss , $\mathrm{pH}$ and color & $400-1100$ & Reflectance & Qiao et al (2007b) \\
\hline & Pork & $\begin{array}{l}\text { Classification and determination of color, } \\
\text { texture and exudation }\end{array}$ & $430-980$ & Reflectance & Qiao et al (2007a) \\
\hline & Pork & Classification and marbling estimation & $430-1000$ & Reflectance & Qiao et al (2007) \\
\hline
\end{tabular}


Table 6. Applications of spectral imaging system in quality evaluation of beef and pork.

\begin{tabular}{|c|c|c|c|c|c|}
\hline Name of Method & $\begin{array}{c}\text { Source of } \\
\text { Meat }\end{array}$ & Application & $\begin{array}{l}\text { Wavelength } \\
\text { range (nm) }\end{array}$ & Imaging mode & References \\
\hline \multirow{14}{*}{$\begin{array}{l}\text { Hyperspectral } \\
\text { imaging technique. }\end{array}$} & Beef & Chemical composition of beef & $900-1700$ & Reflectance & Elmasry G et al (2013) \\
\hline & Beef & Color, $\mathrm{pH}$ and tenderness prediction & $897-1752$ & Reflectance & Elmasry G et al (2012) \\
\hline & Beef & Determination of water holding capacity & $910-1700$ & Reflectance & Elmasry G et al (2011) \\
\hline & Beef & Microbial spoilage detection of beef & $400-1000$ & Reflectance & Peng et al (2011) \\
\hline & Beef & Beef steak tenderness prediction & 496-1036 & Reflectance & Cluff K et al (2008) \\
\hline & Beef & Beef tenderness prediction & $400-1000$ & Reflectance & Naganathan et al (2008 a). \\
\hline & Pork & Marbling detection & $900-1700$ & Reflectance & Huang et al (2014) \\
\hline & Pork & Quantification intramuscular fat content & $900-1700$ & Reflectance & Huang et al (2014) \\
\hline & Pork & Prediction fresh and frozen thawed muscles & $900-1700$ & Reflectance & Barbin et al (2013) \\
\hline & Pork & Microbial contamination in pork & $900-1700$ & Reflectance & Barbin et al (2013) \\
\hline & Pork & Classification and grading of pork & $900-1700$ & Reflectance & Barbin et al (2013) \\
\hline & Pork & Determination marbling score & $460,580,720$ & Reflectance & Liu et al (2012) \\
\hline & Pork & Sensory attributes of pork & $900-1700$ & Reflectance & Barbin et al (2012) \\
\hline & Pork & Categorization of pork quality & $400-1000$ & Reflectance & Liu et al (2010) \\
\hline \multirow{3}{*}{$\begin{array}{l}\text { Hyperspectral } \\
\text { imaging technique. }\end{array}$} & Pork & Prediction total viable bacteria & $400-1100$ & Reflectance & Peng et al (2008) \\
\hline & Lamb & Discrimination of lamb muscle & $910-1700$ & Reflectance & Kamruzzaman et al (2011) \\
\hline & Lamb & Quality attributes of lamb meat & $900-1700$ & Reflectance & Kamruzzaman et al (2011) \\
\hline
\end{tabular}

This is however a ruthless task and is usually not viable due to time and financial concerns (Park et al., 2001). Alternatively, it can be measured by skilled sensory panels or by instrumental methods (Instron testing or other shearing devices) because there is no straight method accessible for predicting tenderness on the real-time manner. Instrumental methods of meat tenderness measurement are sluggish and disparaging. Conversely, sensory analysis with trained panelists is subjective, very costly, time consuming, and also destructive. Therefore, it is necessary to develop a fast, nondestructive, correct, and on-line technique to predict cooked meat tenderness from the new steak. As an objective, consistent, rapid, and spontaneous technique, hyperspectral imaging techniques could be engaged to complete such a task.
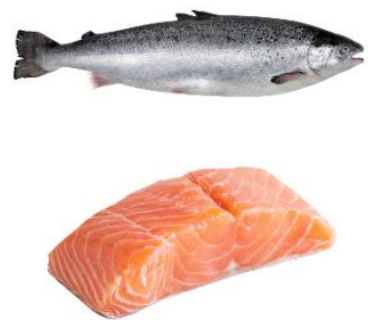

Predicting content of fat, water and salt, EIMasry and Wold, 2008; Segtnan et al., 2009b.

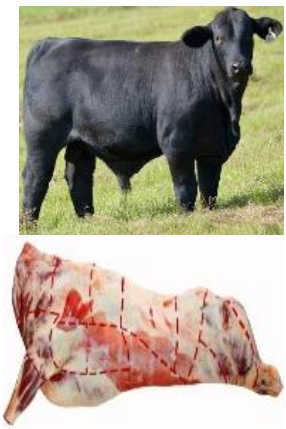

Tenderness identification, Cluff et al., 2008, Peng and Wu, 2008

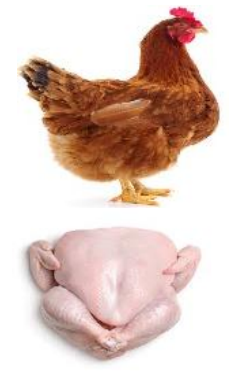

Detection of tumor Knog, 2003, Kong et al., 2004, Nakariyakul Casasent, 2004, 2007
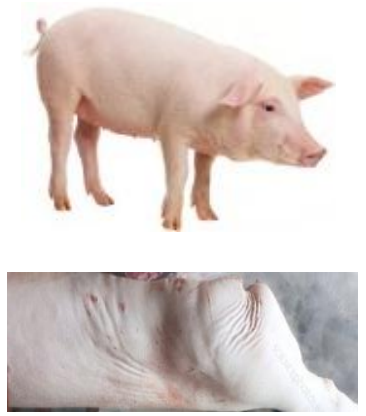

Prediction of quality Qiao et al., 2007a Qiao et al., 2007b Qiao et al., 2007c
Figure 3. Potential application of hyperspectral imaging in quality evaluation of meat (Adapted with permission, G Elmasry et al., 2012).

\section{Conclusion}

Near infrared spectroscopy has substantial prospective to estimate simultaneously numerous physicochemical properties of meat and to classify meat into quality classes. So NIR is an appropriate substitute to laborious, costly, ecologically unfriendly analytical technique. NIR spectroscopy has only restricted capability for evaluating sensory characteristics of meat due to heterogeneity of meat sample. Subsequently, superior NIR calibrations for sensory and technological attributes are essential, chiefly with respect to an improved sampling technique and development of the accuracy of the reference systems. At present time, many researchers and analysts are searching for desirable wavelengths at which NIR measures are more closely connected with the attributes of meat quality. Hyperspectral imaging system is a newly appeared procedure designed to incorporate both optical spectroscopy and customary imaging system. With the advancement of time hyperspectral imaging systems announce innovative inspection services that assist better assessment of meat chemical composition and quality attributes. The current review provides the information regarding hyperspectral imaging system that has the potential ability to fulfill the gap between spectroscopy and imaging techniques by recording full spectrum to each individual pixel in the image. The hyperspectral imaging system can be efficiently used as a dependable and perfect tool for categorize and sorting of different agricultural food products and meat quality assurance and safety authentication. Finally, NIR and hyperspectral imaging system has a huge potential application in detecting chemical composition of meat and diverse quality attributes of meat.

\section{Acknowledgement}

The authors acknowledge the personnel Livestock Production and Management department, Faculty of Veterinary, Animal and Biomedical Sciences, Sylhet Agricultural University, Sylhet-3100, Bangladesh for their valuable assist to prepare the review manuscript.

\section{References}

Alomar D, Gallo C, Castañeda M, \& Fuchslocher R (2003). Chemical and discriminant analysis of bovine meat by near infrared reflectance spectroscopy (NIRS). Meat Sci. 63(4),441-450.

Anderson N M, \& Walker PN (2003). Measuring fat content of ground beef stream using on-line visible/NIR Spectroscopy. Trans ASABE. 46(1), 117-124. 
Anderson S (2007). Determination of fat, moisture, and protein in meat and meat products by using the FOSS FoodScan $^{\mathrm{TM}}$ near-infrared spectrophotometer with FOSS artificial neural network calibration model and associated database: Collaborative study. J. AOAC Int. 90(4),10731083.

Andrés S, Murray I, Navajas EA, Fisher AV, Lambe NR \& Bünger L (2007). Prediction of sensory characteristics of lamb meat samples by near infrared reflectance spectroscopy. Meat Sci.76(3), 509-516.

Balage JM, da Luz E Silva S, Gomide CA, Bonin Mde N, \& Figueira AC (2015). Predicting pork quality using Vis/NIR spectroscopy. Meat Sci.108:37-43.

Barbin D, Elmasry G, Sun DW, \& Allen P (2012). Nearinfrared hyperspectral imaging for grading and classification of pork. Meat Sci. 90(1): 259-268.

Barbin DF, Elmasry G, Sun DW, \& Allen P (2013). Nondestructive determination of chemical composition in intact and minced pork using near-infrared hyperspectral imaging. Food Chem. 138(2-3):1162-1171.

Barbin DF, Elmasry G, Sun DW, Allen P, \& Morsy N (2013). Non-destructive assessment of microbial contamination in porcine meat using NIR hyperspectral imaging. Innov Food Sci Emerg Technol.17, 180-191.

Barbin DF, ElmasryG, Sun DW, \& Allen P (2012). Predicting quality and sensory attributes of pork using near-infrared hyperspectral imaging. Anal. Chim. Acta. 719, 30-42.

Barlocco N, Vadell A, Ballesteros F, Galietta G, \& Cozzolino D (2006). Predicting intramuscular fat, moisture and Warner-Bratzler shear force in pork muscle using near infrared reflectance spectroscopy. Anim Sci. J. 82(1), 111-116.

Berzaghi P, Dalle Zotte A, Jansson LM, \& Andrighetto I (2005). Near-infrared reflectance spectroscopy as a method to predict chemical composition of breast meat and discriminate between different $n-3$ feeding sources. Poult Sci. J. 84(1):128-136.

Büning-Pfaue H (2003). Analysis of water in food by near infrared spectroscopy. Food Chem. 82(1), 107-115.

Čandek-Potokar M, Prevolnik M, \& Skrlep M (2006). Ability of near infrared spectroscopy to predict pork technological traits. J Near Infrared Spectrosc. 14(4), 269-277.

Cozzolino D, Martins V, \& Murray I (2002). Visible and near infrared spectroscopy of beef longissimus dorsi muscle as a means of dicriminating between pasture and corn silage feeding regimes. J Near Infrared Spectrosc. 10(3):187-193.

Cozzolino D, Mattos, D. De, \& Martins, DV (2002). Visible / near infrared reflectance spectroscopy for predicting composition and traching system of production of beef muscle. Anim. SCi. J. 74: 477-484.

Cozzolino D, Murray I, Paterson R., \& Scaife JR (1996). Visible and near infrared reflectance spectroscopy for the determination of moisture, fat and protein in chicken breast and thigh muscle. J Near Infrared Spectrosc. 4(14): 213-223.

Cozzolino D, Murray I, Scaife JR. \& Paterson R (2000). Study of dissected lamb muscles by visible and near infrared reflectance spectroscopy for composition assessment. Anim Sci. J. 70(3):417-423.

De Marchi M, Berzaghi P, Boukha A, Mirisola M., \& Gallo L (2007). Use of near infrared spectroscopy for assessment of beef quality traits. Italian Journal of Anim
Sci. J. 6 (SUPPL. 1), 421-423.

Ding HB, \& Xu RJ (1999). Differentiation of beef and kangaroo meat by visible/near-infrared reflectance spectroscopy. J. Food Sci. 64(5), 814-817.

Du CJ, \& Sun DW (2004). Recent developments in the applications of image processing techniques for food quality evaluation. Trends Food Sci Technol.15(5): 230249.

Elmasry G, Iqbal A, Sun, DW, Allen P, \& Ward P (2011). Quality classification of cooked, sliced turkey hams using NIR hyperspectral imaging system. J. Food Eng. 103(3): 333-344.

ElMasry G, Kamruzzaman M, Sun DW, \& Allen P (2012). Principles and Applications of Hyperspectral Imaging in Quality Evaluation of Agro-Food Products: A Review. Crit Rev Food Sci Nutr. 52(11): 999-1023.

ElMasry G, Sun DW, \& Allen P (2013). Chemical-free assessment and mapping of major constituents in beef using hyperspectral imaging. J. Food Eng.117(2):235246.

ElMasry, G., Sun, D. W., \& Allen, P. (2011). Nondestructive determination of water-holding capacity in fresh beef by using NIR hyperspectral imaging. Food Res Int. 44(9), 2624-2633.

Feng YZ, \& Sun DW (2012). Application of Hyperspectral Imaging in Food Safety Inspection and Control: A Review. Crit Rev Food Sci Nutr. 52(11):1039-1058.

Gaitán-Jurado AJ, Ortiz-Somovilla V, España-España F, Pérez-Aparicio J, \& De Pedro-Sanz, EJ (2008). Quantitative analysis of pork dry-cured sausages to quality control by NIR spectroscopy. Meat Sci.78(4):391-399.

González-Martín I, González-Pérez C, Hernández-Méndez J, \& Alvarez-García N (2003). Determination of fatty acids in the subcutaneous fat of Iberian breed swine by near infrared spectroscopy (NIRS) with a fibre-optic probe. Meat Sci. 65(2):713-719.

Hoving-Bolink, AH., Vedder HW, Merks JWM, De Klein WJH, Reimert, HGM, Frankhuizen, R, Van Den Broek, WHAM, \& Lambooij E (2005). Perspective of NIRS measurements early post mortem for prediction of pork quality. Meat Sci. 69(3): 417-423.

Prevolnik M, Čandek-Potokar M, Škorjanc D, VelikonjaBolta Š, Škrlep M, Žnidaršič T, \& Babnik D (2005). Predicting intramuscular fat content in pork and beef by near infrared spectroscopy. J Near Infrared Spectrosc.13(2):77-85.

Prevolnik M, Škrlep M, Škornjac D, \& Čandek-Potokar M (2010). Application of near infrared spectroscopy to predict chemical composition of meat and meat products. J. Meat. Sci. Technol. 51(2): 133-142.

Prieto N, Andrés S, Giráldez FJ, Mantecón AR, \& Lavín $\mathrm{P}(2006)$. Potential use of near infrared reflectance spectroscopy (NIRS) for the estimation of chemical composition of oxen meat samples. Meat Sci.74 (3): 487496.

Prieto N, López-Campos O, Aalhus JL, Dugan ME, Juárez M, Uttaro B (2014). Use of near infrared spectroscopy for estimating meat chemical composition, quality traits and fatty acid content from cattle fed sunflower or flaxseed. Meat Sci. 98(2):279-88.

Prieto N, Pawluczyk O, Dugan MER, \& Aalhus JN (2017). A review of the principles and application of NearInfreared Spectroscopy to characterize Meat, Fat and Meat products. Appl Spectrosc.71(7):403-1426. 
Qiao J, Wang N, Ngadi MO, Gunenc A, Monroy M, Gariépy C, \& Prasher SO (2007). Prediction of drip-loss, pH, and color for pork using a hyperspectral imaging technique. Meat Sci. 76(1), 1-8.

Ripoll G, Albertí P, Panea B, Olleta JL, \& Sañudo C (2008). Near-infrared reflectance spectroscopy for predicting chemical, instrumental and sensory quality of beef. Meat Sci. 80(3), 697-702.

Rødbotten R, Nilsen BN, \& Hildrum KI (2000). Prediction of beef quality attributes from early post mortem near infrared reflectance spectra. Food Chem. 69(4), 427-436.

Sanderson R, Lister SJ., Dhanoa MS, Barnes RJ, \& Thomas C(1997). Use of near infrared reflectance spectroscopy to predict and compare the composition of carcass samples from young steers. Anim. Sci. J. 65(1): 45-54.

Shaarani S M, Nott KP, \& Hall LD (2006). Combination of NMR and MRI quantitation of moisture and structure changes for convection cooking of fresh chicken meat. Meat Sci. 72(3), (398-403.)
Uddin et al., 2021

Su H, Sha K, Zhang L, Zhang Q, Xu Y, Zhang R, Li H, Sun B. (2014). Development of near infrared reflectance spectroscopy to predict chemical composition with a wide range of variability in beef. Meat Sci. 98(2):110-4.

Tøgersen G, Arnesen JF, Nilsen BN, \& Hildrum, KI (2003). On-line prediction of chemical composition of semifrozen ground beef by non-invasive NIR spectroscopy. Meat Sci. 63(4):515-523.

Tøgersen G, Isaksson T, Nilsen BN, Bakker EA, \& Hildrum KI (1999). On-line NIR analysis of fat, water and protein in industrial scale ground meat batches. Meat Sci. 51(1): 97-102.

VerbekeW, Pérez-Cueto FJA, \& Grunert KG (2011). To eat or not to eat pork, how frequently and how varied? Insights from the quantitative Q-PorkChains consumer survey in four European countries. Meat Sci. 88(4), 619626.

Viljoen M, Hoffman LC, \& Brand TS (2007). Prediction of the chemical composition of mutton with near infrared reflectance spectroscopy. Small Rumin. Res. 69(1-3): 